\title{
Magnetorotational instability in cool cores of galaxy clusters
}

\author{
CARLO NIPOTI ${ }^{1} \dagger$, L. P OSTI I $I^{1}$, S. ET T ORI $I^{2,3}$ \\ A ND M. B I A N C O N I \\ ${ }^{1}$ Dipartimento di Fisica e Astronomia, Università di Bologna, viale Berti-Pichat 6/2, I-40127 \\ Bologna, Italy \\ ${ }^{2}$ INAF, Osservatorio Astronomico di Bologna, via Ranzani 1, I-40127 Bologna, Italy \\ ${ }^{3}$ INFN, Sezione di Bologna, viale Berti-Pichat 6/2, I-40127 Bologna, Italy \\ ${ }^{4}$ Institute of Astro and Particle Physics, University of Innsbruck, A-6020 Innsbruck, Austria
}

(Received in original form March 17 2015. Accepted May 29 2015)

Clusters of galaxies are embedded in halos of optically thin, gravitationally stratified, weakly magnetized plasma at the system's virial temperature. Due to radiative cooling and anisotropic heat conduction, such intracluster medium (ICM) is subject to local instabilities, which are combinations of the thermal, magnetothermal and heat-flux-driven buoyancy instabilities. If the ICM rotates significantly, its stability properties are substantially modified and, in particular, also the magnetorotational instability (MRI) can play an important role. We study simple models of rotating cool-core clusters and we demonstrate that the MRI can be the dominant instability over significant portions of the clusters, with possible implications for the dynamics and evolution of the cool cores. Our results give further motivation for measuring the rotation of the ICM with future $\mathrm{X}$-ray missions such as ASTRO-H and ATHENA.

\section{PACS codes:}

\section{Introduction}

Galaxy clusters are embedded in gaseous halos at the system's virial temperature $\left(10^{7}-10^{8} \mathrm{~K}\right)$. Such intracluster medium (ICM) is a dilute, weakly magnetized plasma, stratified in the cluster gravitational potential. The question of the local stability of the ICM is a fundamental piece in the puzzle of the evolution of clusters and in particular of cool-core clusters, in which the cooling time of the plasma in the central regions is shorter than the cluster age. The results of linear-stability analysis and magnetohydrodynamics (MHD) simulations indicate that, due to the joint effect of radiative cooling and anisotropic heat conduction, the ICM is subject to several local instabilities, which are combinations of the thermal instability (TI; Field 1965), the magnetothermal instability (MTI; Balbus 2000) and the heat-flux-driven buoyancy instability (HBI; Quataert 2008). Cosmological hydrodynamic simulations suggest that the X-ray emitting halos of clusters, though mainly pressure supported, might rotate significantly (Fang et al. 2009; Lau et al. 2012), with a contribution of rotation especially important in the cool cores of relaxed clusters (Nagai et al. 2013). Observationally, the most direct signature of ICM rotation would be the spatially resolved measure of shifted X-ray emission line centroids. Unfortunately, given the relatively poor spectral resolution $(\gtrsim 100 \mathrm{eV})$

$\dagger$ Email address for correspondence: carlo.nipoti@unibo.it 
of currently available X-ray instruments, so far there are no meaningful constraints on rotation based on such a measure, which requires the spectral capabilities of the up-coming X-ray calorimeters on board of ASTRO-H (Takahashi et al. 2014; Kitavama et al. 2014) and ATHENA (Ettori et al. 2013). Spatially unresolved rotation contributes, together with the turbulence, to the emission line broadening: the present observational measures of the X-ray emission line widths (Sanders \& Fabian 2013; Pinto et al. 2015), even when combined with measures of the flattening of the X-ray isophotes, leave ample room for rotational motions (Bianconi et al. 2013, hereafter BEN13). If the ICM rotates, its stability properties are substantially modified (Balbus 2001; Nipoti 2010) and, in particular, also the magnetorotational instability (MRI; Balbus \& Hawlev 1991; see also Velikhov 1959 and Chandrasekhar 1960) can play an important role (Nipoti \& Posti 2014, hereafter NP14).

The linear evolution of local axisymmetric perturbations in a stratified, rotating, radiatively cooling, weakly magnetized plasma is determined by the dispersion relation derived in Nipoti \& Posti (2013, hereafter NP13). NP14 studied the nature of the instabilities of such a plasma in two illustrative cases, generically representative of the physical conditions in galactic and cluster atmospheres. Here we focus on the cool cores of galaxy clusters and we discuss the stability properties of rotating cool-core models built on the basis of the work of BEN13. The present study extends the results of NP14, as here we study the nature and linear growth rate of the plasma instabilities throughout observationally motivated cool-core cluster models. In this work we limit ourselves to axisymmetric perturbations, but the results of NP14 suggest that the study of nonaxisymmetric disturbances should lead to similar conclusions. Though we consider global cluster models, we study only the stability against local perturbations, i.e. disturbances with sizes much smaller than the characteristic scale-lengths of the system. In addition to the local instabilities found in the present study, the ICM could be subject to global unstable modes (e.g. Latter \& Kunz 2012), which by construction would elude our analysis.

The paper is organized as follows. The governing MHD equations are given in Section 2 , the unperturbed models are presented in Section 3 and the dispersion relation obtained from the linear-stability analysis is given in Section 4. Section 5 describes the properties of the local instabilities occurring in the cluster models and Section [6] concludes.

\section{Governing equations}

A stratified, rotating, magnetized, dilute plasma in the presence of thermal conduction and radiative cooling is governed by the following MHD equations:

$$
\begin{aligned}
& \frac{\partial \rho}{\partial t}+\nabla \cdot(\rho \mathbf{v})=0, \\
& \rho\left[\frac{\partial \mathbf{v}}{\partial t}+(\mathbf{v} \cdot \nabla) \mathbf{v}\right]=-\nabla\left(p+\frac{B^{2}}{8 \pi}\right)-\rho \nabla \Phi+\frac{1}{4 \pi}(\mathbf{B} \cdot \nabla) \mathbf{B}, \\
& \frac{\partial \mathbf{B}}{\partial t}-\nabla \times(\mathbf{v} \times \mathbf{B})=0, \\
& \frac{p}{\gamma-1}\left[\frac{\partial}{\partial t}+\mathbf{v} \cdot \nabla\right] \ln \left(p \rho^{-\gamma}\right)=-\nabla \cdot \mathbf{Q}-\rho \mathcal{L}+\mathcal{H},
\end{aligned}
$$

with the additional condition $\nabla \cdot \mathbf{B}=0$. Here $\rho, p, T, \mathbf{v}$ and $\mathbf{B}$ are, respectively, the density, pressure, temperature, velocity and magnetic field of the fluid, $\Phi$ is the external gravitational potential (we neglect self-gravity), $\gamma=5 / 3$ is the adiabatic index, $\mathcal{H}$ is the 
heating rate per unit volume, $\mathcal{L}=\mathcal{L}(T, \rho)$ is the radiative energy loss per unit mass of fluid, and

$$
\mathbf{Q}=-\frac{\chi \mathbf{B}(\mathbf{B} \cdot \nabla) T}{B^{2}}
$$

is the conductive heat flux, where $\chi \equiv \kappa T^{5 / 2}$ is Spitzer (1962) electron conductivity with $\kappa \simeq 1.84 \times 10^{-5}(\ln \Lambda)^{-1} \mathrm{erg} \mathrm{s}^{-1} \mathrm{~cm}^{-1} \mathrm{~K}^{-7 / 2}$ and $\ln \Lambda$ is the Coulomb logarithm. Neglecting the weak temperature and density dependence of $\ln \Lambda$, here we fix $\ln \Lambda=30$, so $\kappa$ is a constant and $\chi=\chi(T) \propto T^{5 / 2}$. As we consider rotating fluids, we work in cylindrical coordinates $(R, \phi, z)$, where $R=0$ is the rotation axis. The governing equations (2.12.4) are given explicitly in cylindrical coordinates in Appendix A .

The expression (2.5) for $\mathbf{Q}$ accounts for the fact that in a dilute magnetized plasma heat is significantly transported by electrons only along the magnetic field lines. For simplicity, even if the medium is magnetized, we have assumed that the pressure is isotropic, neglecting the fact that momentum transport is anisotropic in the presence of a magnetic field (i.e. the so-called Braginskii viscosity; Braginskii 1965). Though this approximation is not rigorously justified, we adopt it in the working hypothesis that anisotropic pressure is not the crucial factor in determining the dominant instabilities of a rotating ICM. The importance of the effects of the Braginskii viscosity on plasma stability is debated (Kunz 2011; Latter \& Kunz 2012; Parrish et al. 2012), so the limits of this approximation must be taken into account when interpreting the results of the present work.

The term $\mathcal{H}$ in equation (2.4) represents an unspecified source of heating that is expected to counteract radiative cooling and prevent a cooling catastrophe. There is general consensus that heat conduction cannot be entirely responsible for halting cooling flows (e.g. Parrish et al. 2009) and that other sources of heating, such as feedback from the central active galactic nucleus (AGN), must be at work to balance cooling in a timeaveraged sense. In the absence of physically motivated and analytically tractable models of such a heating term, here we just consider a toy model in which, for a given cluster model, $\mathcal{H}$ is a fixed position-dependent function (for a discussion see McCourt et al. 2012): the main results of this work are independent of this approximation, though very crude.

\section{Cool-core cluster models}

We consider here three rotating cool-core cluster models (named C1, C2 and C3), which are slightly modified versions of those presented in BEN13 (indicated as CC1, CC2 and CC3 in that paper). In all these models, which represent massive galaxy clusters, the total gravitational potential $\Phi$ is given by a spherica $\$$ Navarro-Frenk-White (NFW) model (Navarro et al. 1995) with virial mass $M_{200}=10^{15} M_{\odot}$, scale radius $r_{\mathrm{s}}=519 \mathrm{kpc}$ and virial radius $r_{200}=2066 \mathrm{kpc}$. The plasma rotates differentially with azimuthal velocity $v_{0 \phi}=\Omega R$, where $\Omega=\Omega(R)$ is the angular velocity and the rotation law is different in the three models (see section 2.3 of BEN13). The other velocity components are null. Assuming that the ICM magnetic field is dynamically unimportant, we construct the unperturbed pressure $p_{0}=p_{0}(R, z)$, density $\rho_{0}=\rho_{0}(R, z)$ and temperature $T_{0}=T_{0}(R, z)$ fields as axisymmetric stationary solutions of equations (2.1]2.2) with $\mathbf{B}=0$. As $\Omega$ does not depend on $z$, the gas distribution is barotropic $\left(\rho_{0}, p_{0}\right.$ and $T_{0}$ are stratified on

$\dagger$ In a more realistic model the total gravitational potential would be axisymmetric or triaxial. Though idealized, a model with spherical potential is an interesting limiting case because the flattening of the ICM distribution is entirely due to rotation. 


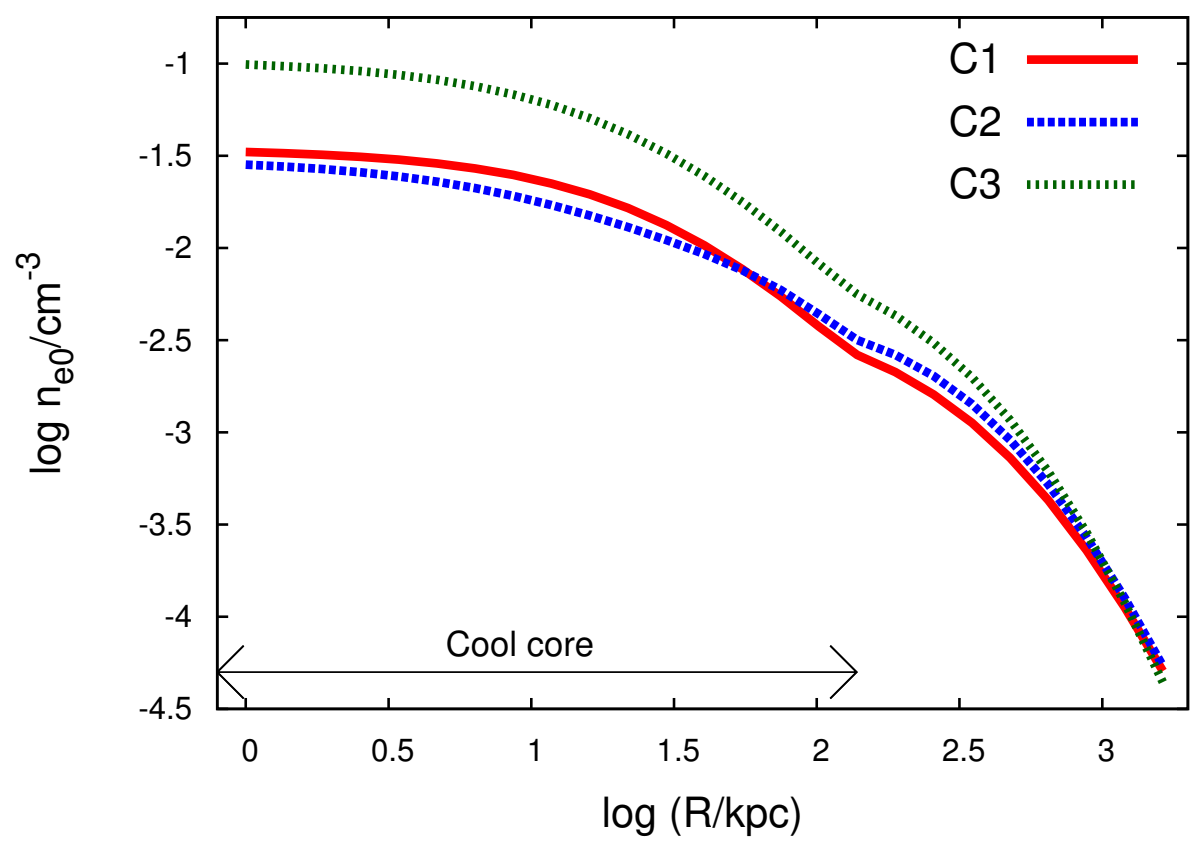

FIGURE 1. ICM electron number density profiles in the equatorial plane of the rotating cool-core cluster models C1, C2 and C3 (see Section 3).

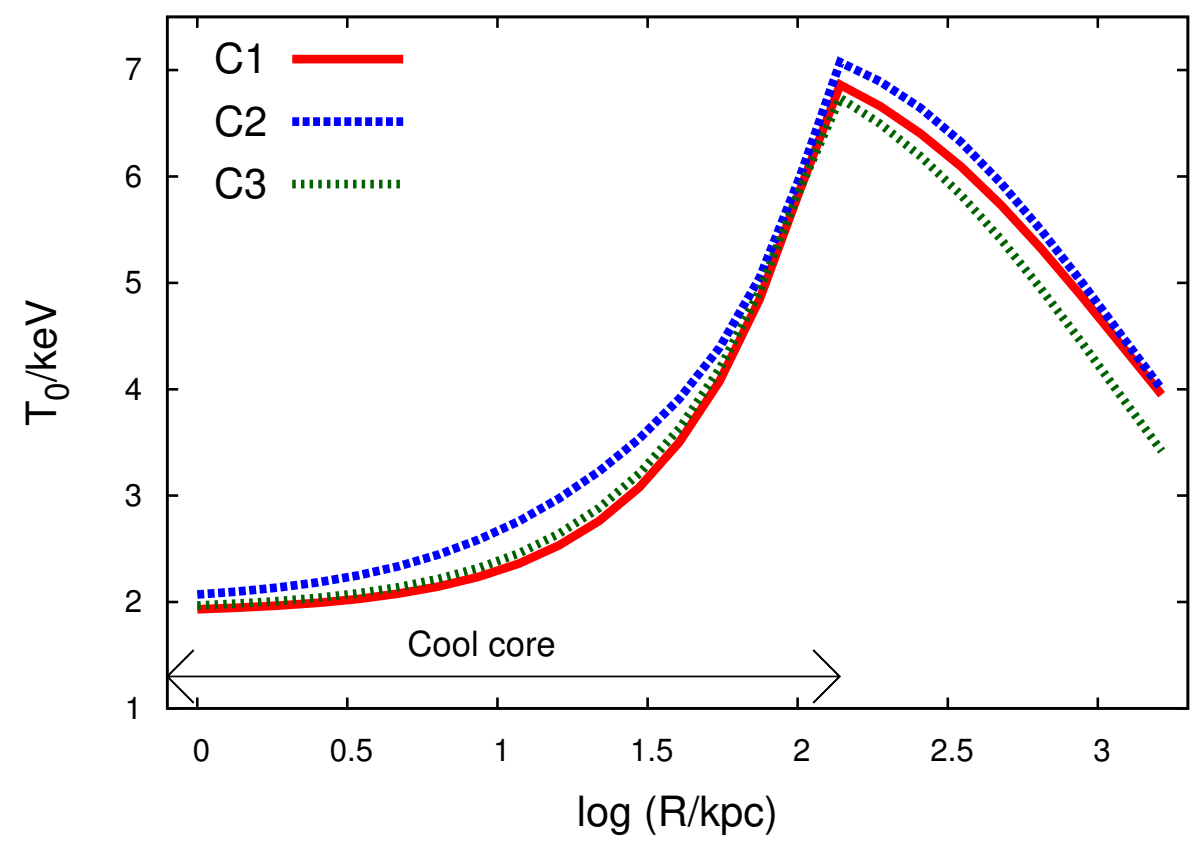

FiguRE 2. ICM temperature profiles in the equatorial plane of the same models as in Fig. 1 


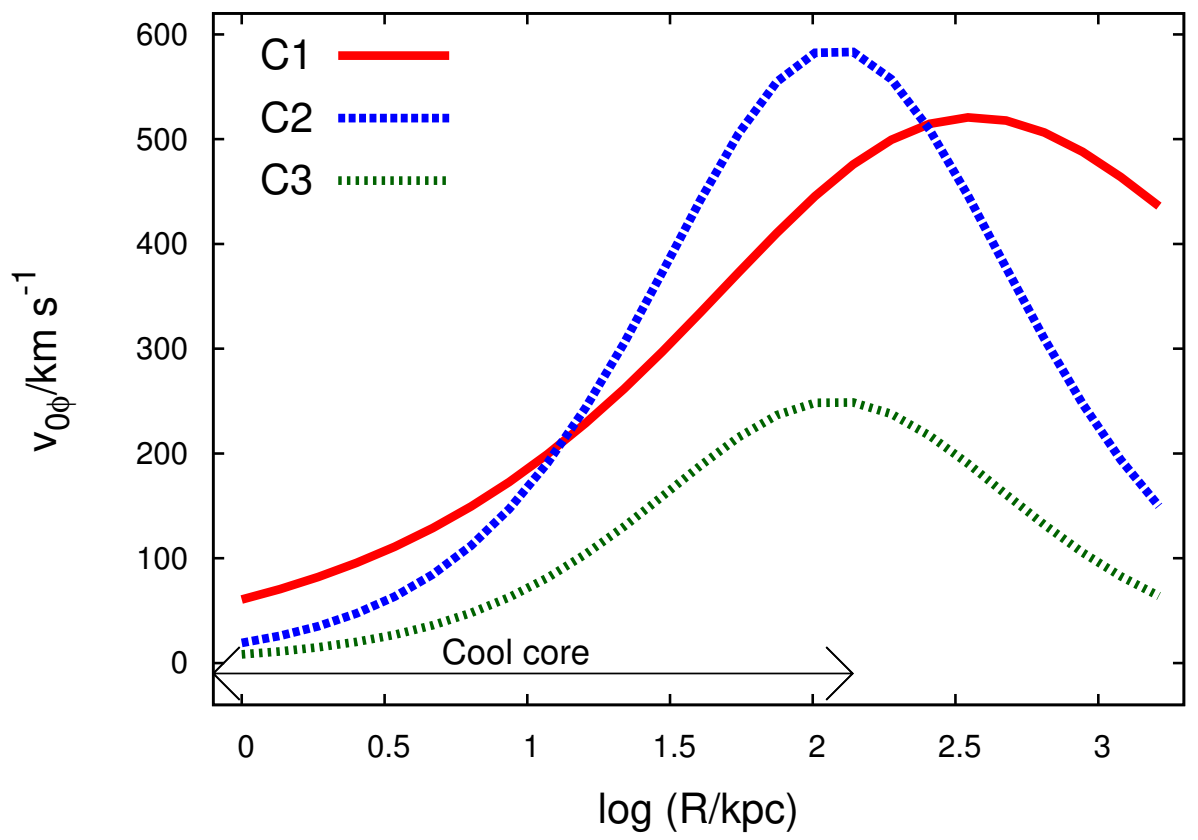

FIGURE 3. ICM rotation speed as a function of radius for the same models as in Fig. 1.

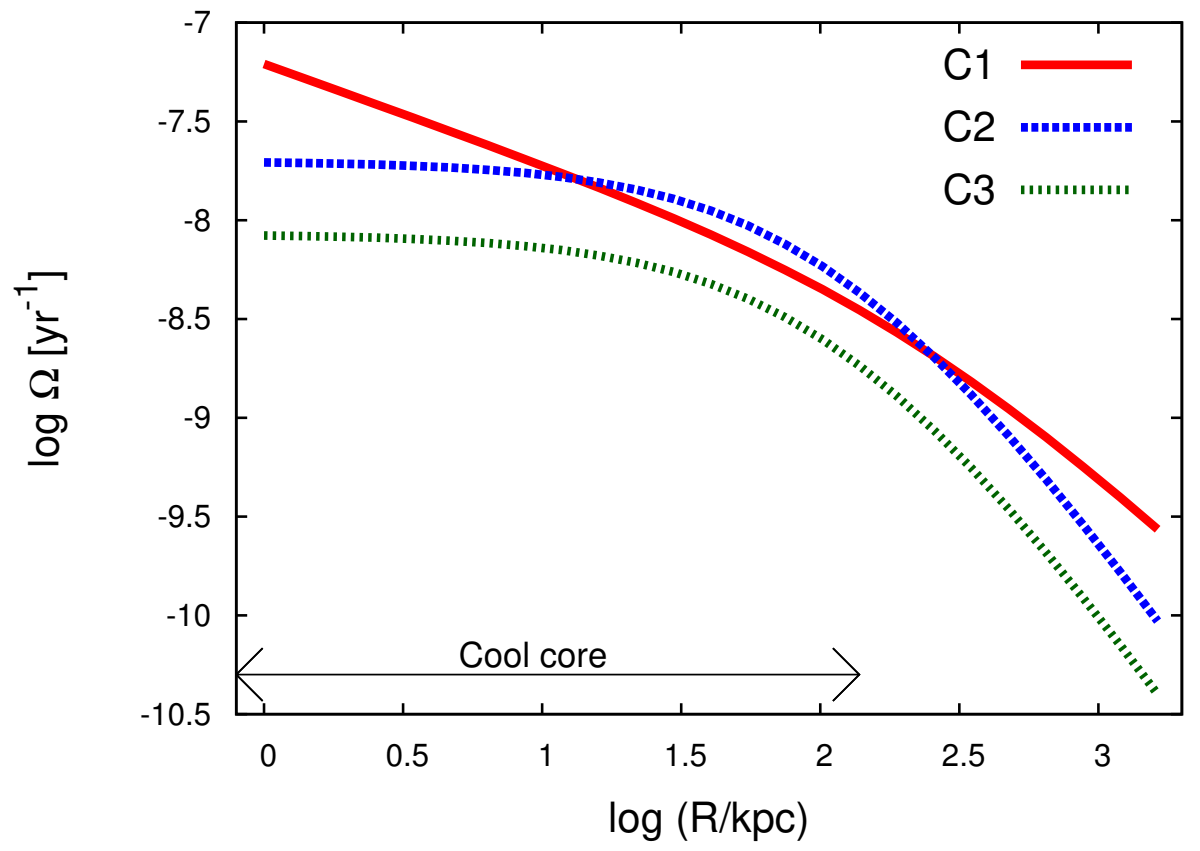

FIGURE 4. ICM angular velocity as a function of radius for the same models as in Fig. 1. 


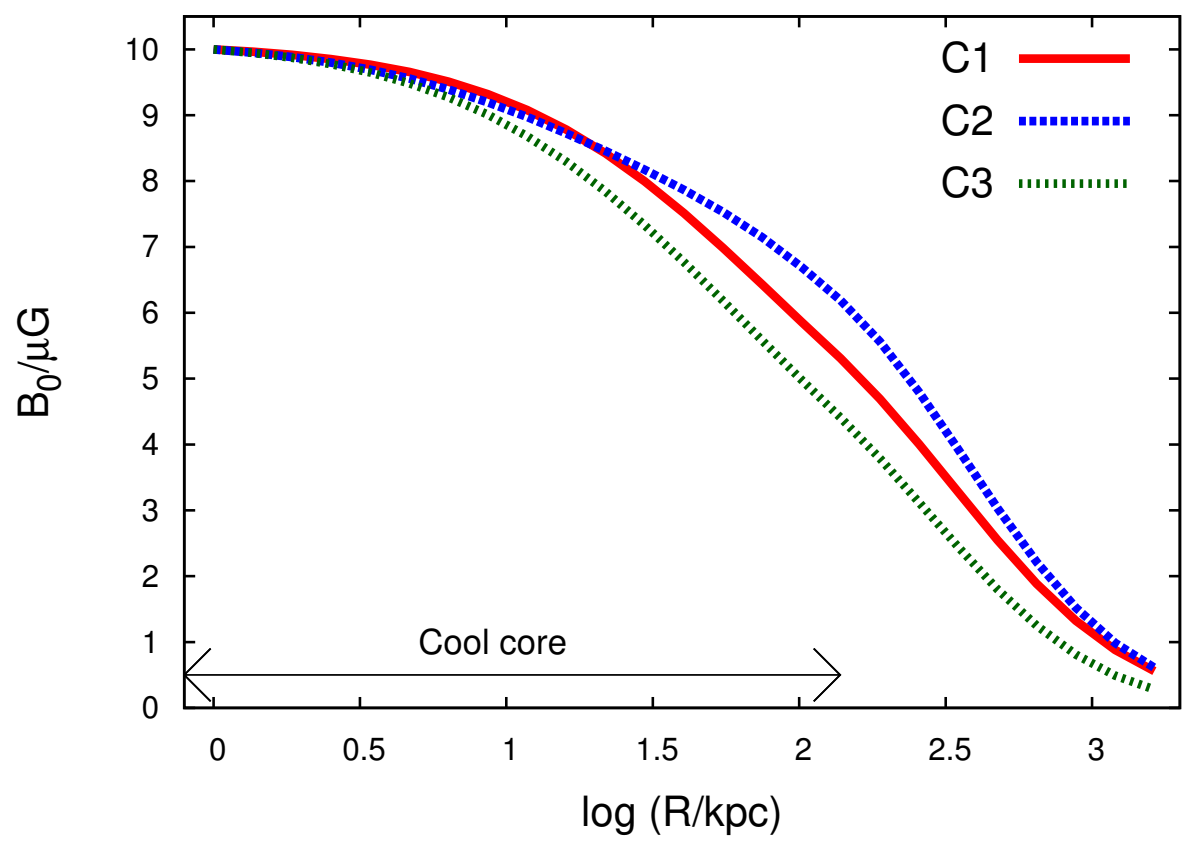

FIGURE 5. ICM magnetic field modulus as a function of radius in the equatorial plane of the same models as in Fig. 1]

the surfaces of constant effective potential). In particular, the plasma is modeled as an equilibrium two-component composite polytrope with outer polytropic index $\tilde{\gamma}_{\text {out }}=1.14$ and inner polytropic index $\tilde{\gamma}_{\text {in }}<1$, so the ICM temperature decreases outwards in the outer regions and increases outwards in the inner regions (cool core). The parameters of models C1, C2 and C3 are, respectively, the same as those of models CC1, CC2 and CC3 of BEN13, with the only exception of the inner polytropic index $\tilde{\gamma}_{\text {in }}$, which is $\tilde{\gamma}_{\text {in }}=0.49$ in model $\mathrm{C} 1, \tilde{\gamma}_{\text {in }}=0.43$ in model $\mathrm{C} 2, \tilde{\gamma}_{\text {in }}=0.56$ in model C3 (these values, smaller than in BEN13, lead to higher central densities and lower central temperatures). The plasma temperature is in the range $2-7 \mathrm{keV}$ and the size of the cool core is about $140 \mathrm{kpc}$ in the equatorial plane. As in BEN13, we assume that the ICM metallicity is everywhere $3 / 10$ of the solar metallicity. Figs. 1,4 plot the radial profiles, in the equatorial plane, of electron number density $n_{e 0}$, temperature $T_{0}$, rotation speed $v_{0 \phi}$ and angular velocity $\Omega$. As discussed in BEN13, the above models of rotating clusters are realistic, in the sense that they are consistent with the current measurements of the ellipticity of the $\mathrm{X}$-ray isophotes and of the width of the X-ray emission lines. Additional constraints on the rotation of the ICM derive from the so-called hydrostatic mass bias, that is the discrepancy between cluster mass estimates from gravitational lensing and those based on the assumption that the ICM is in hydrostatic equilibrium. In Appendix B we show that models $\mathrm{C} 1, \mathrm{C} 2$ and $\mathrm{C} 3$ are realistic also in this respect.

Given a cluster model, we assume that the heating term $\mathcal{H}=\mathcal{H}(R, z)$ appearing in the energy equation (2.4) is such that $\mathcal{H}-\rho_{0} \mathcal{L}\left(T_{0}, \rho_{0}\right)-\nabla \cdot \mathbf{Q}_{0}=0$, where $\mathbf{Q}_{0}$ is the unperturbed conductive heat flux. We remark that in our models $\mathcal{H}$ is not a function of the hydrodynamic variables, but depends explicitly on the coordinates $R$ and $z$. To complete our cluster models we must specify the properties of the unperturbed magnetic field $\mathbf{B}_{0}$, which, though not relevant to the equilibrium configuration (because 
$\left.\beta \equiv 8 \pi p_{0} / B_{0}^{2} \gg 1\right)$, influences the stability of the ICM. We do not attempt a full characterization of the magnetic field throughout the global cluster model. For simplicity, in this work we focus only on the equatorial plane $z=0$, and we specify locally $\mathbf{B}_{0}$ as a function of cylindrical radius $R$. The stationary unperturbed magnetic field must satisfy Ferraro (1937) isorotation law $\mathbf{B}_{0} \cdot \nabla \Omega=0$, so $B_{0 R}=0$ in the considered barotropic distributions. For each cluster model, we consider two cases: one in which the only nonvanishing component of the unperturbed magnetic field is $B_{0 z}$ (so $B_{0 \phi}=0$ ) and another in which both $B_{0 z}$ and $B_{0 \phi}$ are non-vanishing $\left(B_{0 \phi} / B_{0 z}=10\right)$. In all cases, given the unperturbed pressure distribution $p_{0}$, the unperturbed magnetic field modulus is fixed by imposing a constant value of $\beta$ such that at the centre $B_{0}=10 \mu \mathrm{G}$ (consistent with observational estimates; e.g. Brüggen 2013), i.e. $\beta=29.3$ for model $\mathrm{C} 1, \beta=26.8$ for model $\mathrm{C} 2$ and $\beta=89$ for model C3. The profiles of the magnetic field modulus for the three models are shown in Fig. 5. We note that in the equatorial plane the unperturbed conductive heat flux $\mathbf{Q}_{0}$ vanishes, because at $z=0$ the magnetic field lines are isothermal (the only non vanishing component of the temperature gradient is the radial one, which, combined with $B_{0 R}=0$, implies $\mathbf{Q}_{0}=0$ ), so the condition of energy balance is simply $\mathcal{H}=\rho_{0} \mathcal{L}\left(T_{0}, \rho_{0}\right)$.

\section{Linear stability analysis}

The stability analysis is performed by linearizing the system (2.1)2.4) with local axisymmetric Eulerian perturbations of the form $f \mathrm{e}^{-\mathrm{i} \omega t+\mathrm{i} k_{R} R+\mathrm{i} k_{z} z}$ with $|f| \ll\left|f_{0}\right|$, where $f_{0}$ is the unperturbed quantity, $\omega$ is the perturbation frequency, and $k_{R}$ and $k_{z}$ are, respectively, the radial and vertical components of the perturbation wave-vector $\mathbf{k}$. In the short wave-length and low frequency approximation, the resulting dispersion relation for $n \equiv-\mathrm{i} \omega$ is $(\mathrm{NP} 13)$

$$
\begin{aligned}
n^{5}+ & \omega_{\mathrm{d}} n^{4}+\left[\omega_{\mathrm{BV}}^{2}+\omega_{\text {rot }}^{2}+2 \omega_{\mathrm{A}}^{2}\right] n^{3}+\left[\left(\omega_{\mathrm{rot}}^{2}+2 \omega_{\mathrm{A}}^{2}\right) \omega_{\mathrm{d}}+\omega_{\mathrm{A}}^{2} \omega_{\mathrm{c}, \mathrm{mag}}\right] n^{2} \\
& +\omega_{\mathrm{A}}^{2}\left(\omega_{\mathrm{A}}^{2}+\omega_{\mathrm{BV}}^{2}+\omega_{\text {rot }}^{2}-4 \Omega^{2} \frac{k_{z}^{2}}{k^{2}}+\omega_{\mathrm{c}, \phi}^{2}\right) n \\
& +\omega_{\mathrm{A}}^{2}\left[\left(\omega_{\mathrm{A}}^{2}+\omega_{\text {rot }}^{2}-4 \Omega^{2} \frac{k_{z}^{2}}{k^{2}}\right) \omega_{\mathrm{d}}+\omega_{\mathrm{A}}^{2} \omega_{\mathrm{c}, \mathrm{mag}}\right]=0,
\end{aligned}
$$

where the quantities indicated with subscripted $\omega$ are characteristic frequencies related to local properties of the unperturbed distribution and of the wave-vector. Specifically, $\omega_{\mathrm{d}} \equiv$ $\omega_{\text {th }}+\omega_{c, a}$, where $\omega_{\text {th }}$ is the TI frequency and $\omega_{c, a}$ is the anisotropic thermal conduction frequency, $\omega_{\mathrm{BV}}$ is the Brunt-Väisälä frequency, $\omega_{\text {rot }}$ is the angular momentum gradient frequency, $\omega_{\mathrm{A}}$ is the Alfvén frequency, and $\omega_{\mathrm{c} \text {,mag }}$ and $\omega_{\mathrm{c}, \phi}$ are two other frequencies associated with thermal conduction mediated by the magnetic field (all the definitions are given in NP13). The radiative energy loss per unit mass of fluid $\mathcal{L}\left(\rho_{0}, T_{0}\right)$, appearing in $\omega_{\text {th }}$ (see NP13), is computed using the collisional ionization equilibrium cooling function of Sutherland \& Dopita (1993). In the dispersion relation (4.1) there are no terms related to the heating source $\mathcal{H}$ appearing in equation (2.4), because we are considering Eulerian perturbations at a fixed point in space and $\mathcal{H}$ is a function of position, but not of the hydrodynamic variables. For fixed wave-vector k, given a cool-core cluster model (see Section 3), the coefficients of equation (4.1) are fully determined, so the problem of the local linear stability of the plasma is reduced to finding the roots of a polynomial equation. 


\section{Results}

For each of the considered cluster models, at different radii $R$ in the equatorial plane, for given wave-vector $\mathbf{k}$ we numerically computed the solutions of the dispersion relation (4.1) using the IDL (Interactive Data Language) routine FZ_Roots (as in NP14). Depending on the signs of their real $\left(\mathrm{R}_{n}\right)$ and imaginary $\left(\mathrm{I}_{n}\right)$ parts, the solutions are classified as stable $\left(\mathrm{R}_{n} \leqslant 0\right)$, overstable $\left(\mathrm{R}_{n}>0, \mathrm{I}_{n} \neq 0\right)$ or monotonically unstable $\left(\mathrm{R}_{n}>0, \mathrm{I}_{n}=0\right)$ modes. As overstable linear disturbances are unlikely to enter the non-linear regime (Malagoli et al. 1987; Binnev et al. 2009), here we focus only on the more interesting monotonically unstable linear modes. For each model we compute, as a function of radius $R$, the growth rate $n_{\text {inst }}$ of the fastest-growing monotonically unstable mode. At each radius, $n_{\text {inst }}$ is calculated considering modes with wave-number $k=\sqrt{k_{R}^{2}+k_{z}^{2}}$ in the range $10<k h<1000$, where $h \equiv(|\nabla p| / p)^{-1}$ is the pressure scale-height, which depends on $R$ : this guarantees that the perturbation wave-length is short with respect to the characteristic scales of the system at all radii. The radial-to-vertical wave-number ratio $k_{R} / k_{z}$ is allowed to vary in the range $-25 \leqslant k_{R} / k_{z} \leqslant 25$.

As discussed in NP13 and NP14, in general the instabilities of rotating, weakly magnetized, radiatively cooling plasmas arise from combinations of TI, MTI, HBI and MRI. In order to understand the physical consequences of the instability it is important to determine the nature of the dominant unstable modes. NP14 have shown that, though the different modes are entangled, it is possible to identify the different branches of the solutions in terms of combinations of well-known modes (TI, MRI, MTI, HBI, rotation, buoyancy and Alfvén modes). In practice, this is done by inspecting the behaviour and the growth rates of the individual branches of the solutions as functions of the wavenumber and comparing the results with those obtained for simpler configurations (for instance in the absence of radiative cooling, rotation or magnetic field). Applying this technique to the cluster models here considered, we have been able to identify the nature of the dominant instabilities at different radii.

It is useful to compare the maximum instability growth rate $n_{\text {inst }}$ with the characteristic rates of radiative cooling and of the MRI. Therefore, we define the cooling rate

$$
n_{\text {cool }} \equiv \frac{\gamma-1}{\gamma} \frac{\mu m_{\mathrm{p}} \mathcal{L}\left(T_{0}, \rho_{0}\right)}{k_{\mathrm{B}} T_{0}},
$$

which for each unperturbed model is a function of position (here $\mu=0.59$ is the mean mass per particle in units of the proton mass $m_{\mathrm{p}}$ ). The MRI growth rate $n_{\mathrm{MRI}}$ is the maximum growth rate of monotonically unstable modes that are solutions of the dispersion relation (Balbus \& Hawley 1991)

$$
n^{4}+\left[\omega_{\mathrm{BV}}^{2}+\omega_{\text {rot }}^{2}+2 \omega_{\mathrm{A}}^{2}\right] n^{2}+\omega_{\mathrm{A}}^{2}\left(\omega_{\mathrm{A}}^{2}+\omega_{\mathrm{BV}}^{2}+\omega_{\text {rot }}^{2}-4 \Omega^{2} \frac{k_{z}^{2}}{k^{2}}\right)=0,
$$

which is obtained from the more general dispersion relation (4.1) in the limit of absence of radiative cooling and thermal conduction $\left(\omega_{\mathrm{d}}=0, \omega_{\mathrm{c}, \mathrm{mag}}=0, \omega_{\mathrm{c}, \phi}=0\right)$. As well known, the TI growth rate $\left|\omega_{\text {th }}\right|$ is of the order of, but typically somewhat higher than the cooling rate $n_{\text {cool }}$, which is at the basis of the existence of the TI in astrophysical plasmas (Field 1965). The MRI growth rate $n_{\mathrm{MRI}}$ is proportional to the plasma angular velocity $\Omega$ (Balbus \& Hawley 1991).

As mentioned in Section 3 for each cluster model, we consider two cases: one in which $B_{0 \phi}=0$ and another in which $B_{0 \phi} / B_{0 z}=10$ at all radii. The only difference between these two families of model is the value of the ratio $B_{0 \phi} / B_{0 z}$ : all the other properties are the same, including the radial profile of the magnetic field modulus, which is shown in Fig. 囵. 


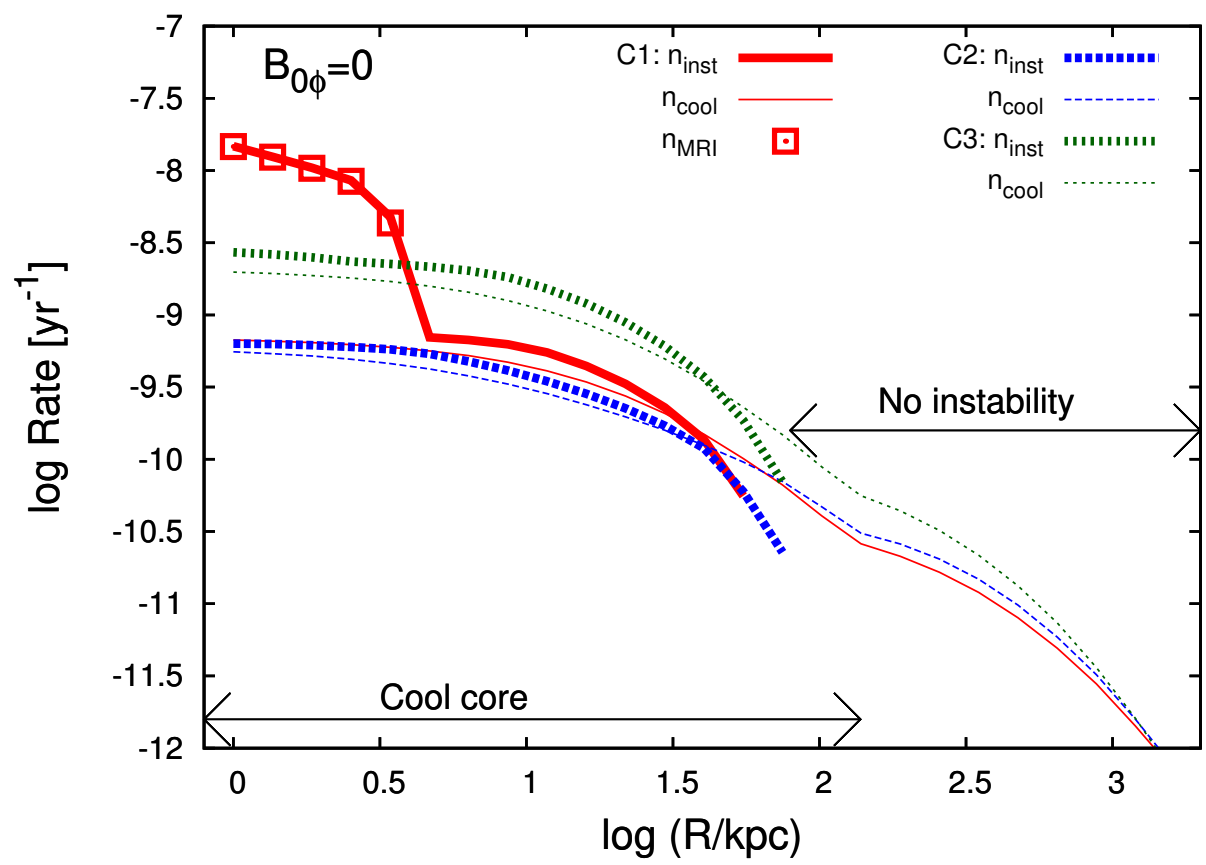

FIGURE 6. Maximum instability growth rate $n_{\text {inst }}$, cooling rate $n_{\text {cool }}$, and MRI growth rate $n_{\text {MRI }}$ as functions of radius in the equatorial plane of the same models as in Fig. 11 Here $B_{0 \phi}=B_{0 R}=0$ and $B_{0 z} \neq 0$. At $R \gtrsim 80 \mathrm{kpc}$ there are no monotonically unstable modes, at least in the explored wave-vector space.

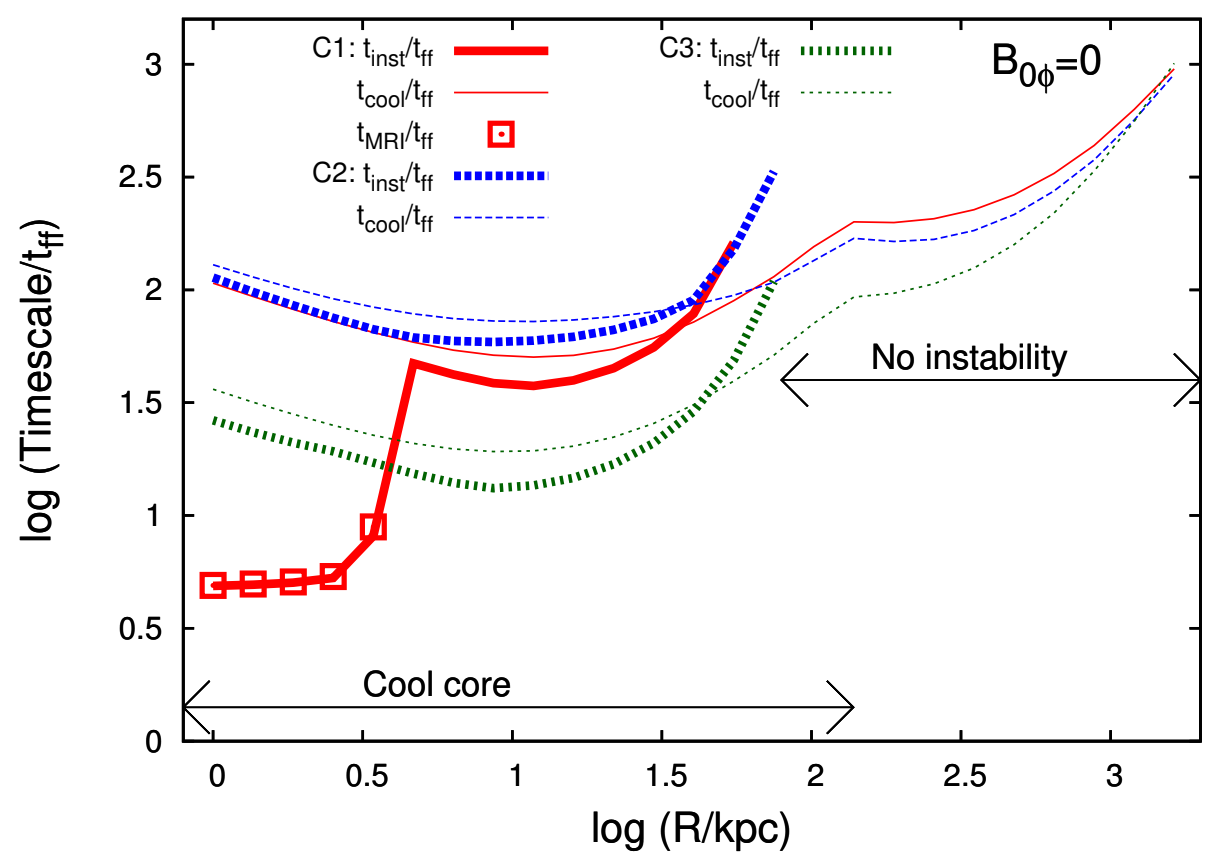

FiguRE 7 . Instability $\left(t_{\text {inst }}\right)$, cooling $\left(t_{\text {cool }}\right)$ and MRI $\left(t_{\mathrm{MRI}}\right)$ timescales normalized to the free-fall time $t_{\mathrm{ff}}$ as functions of radius in the equatorial plane of the same models as in Fig. 6 


\subsection{Models with vanishing azimuthal magnetic field $\left(B_{0 \phi}=0\right)$}

Fig. 6 plots, as a function of radius, the maximum growth rate $n_{\text {inst }}$ in the equatorial plane of models $\mathrm{C} 1, \mathrm{C} 2$ and $\mathrm{C} 3$ when $B_{0 \phi}=0$. For comparison, the cooling $\left(n_{\text {cool }}\right)$ and MRI $\left(n_{\text {MRI }}\right)$ rates are also plotted for the same models. All models are unstable in their cool core, while no monotonically unstable modes are found at $R \gtrsim 80 \mathrm{kpc}$. Model C1 is unstable for MRI in the inner $\sim 10 \mathrm{kpc}$ (instability timescale $\sim 10^{8} \mathrm{yr}$ ), while at larger radii the system is unstable for TI (instability timescale $\sim 10^{9} \mathrm{yr}$, of the order of the cooling time). Models C2 and C3 are not unstable for MRI, but present TI modes throughout the cool core with instability timescales between $5 \times 10^{8}$ and $5 \times 10^{9} \mathrm{yr}$. Together with the absolute timescales, it is also useful to consider the timescales normalized to the local dynamical ("free-fall") time $t_{\mathrm{ff}} \equiv[2 r / g(r)]^{1 / 2}$, where $g=\mathrm{d} \Phi / \mathrm{d} r$ is the modulus of the gravitational force and $r$ is the radial spherical coordinate. Fig. 7 plots, as functions of radius, the instability $\left(t_{\text {inst }} \equiv n_{\text {inst }}^{-1}\right)$, cooling $\left(t_{\text {cool }} \equiv n_{\text {cool }}^{-1}\right)$ and $\operatorname{MRI}\left(t_{\mathrm{MRI}} \equiv n_{\mathrm{MRI}}^{-1}\right)$ timescales normalized to $t_{\mathrm{ff}}$. It is apparent that, when present, the MRI grows on timescales as short as $5 t_{\mathrm{ff}}$, while the TI timescale is typically $10-100 t_{\mathrm{ff}}$.

Though all models satisfy at all radii the necessary condition to have MRI $\left(B_{0 z} \neq 0\right.$ and $\mathrm{d} \Omega / \mathrm{d} R<0$; see Fig. 4), MRI modes are present only in the inner core of model $\mathrm{C} 1$. We recall that the MRI occurs only for wave-numbers smaller than a critical wavenumber $k_{\mathrm{MRI}}$, which, with the exception of the centre of model $\mathrm{C} 1$, is outside the explored wave-number interval (in other words, short wave-length perturbations are not unstable for MRI in these cases). Neither the HBI nor the MTI contributes to the unstable modes: these instabilities are related to anisotropic thermal conduction and can occur when the temperature either increases (HBI) or decreases (MTI) outwards. In the cool core the temperature increases outwards, but the magnetic field lines are isothermal so no HBI is expected. Out of the cool core the temperature decreases outwards, so in principle the MTI might occur, but in fact no MTI modes are found in the explored wave-vector space. Similarly to the MRI, the MTI occurs only for wave-numbers smaller than a critical wavenumber $k_{\text {MTI }}$ (Balbus 2000), which is out of the explored wave-number interval (as it happens for the MRI, short wave-length disturbances are not unstable for MTI in these cases).

\subsection{Models with dominant azimuthal magnetic field $\left(B_{0 \phi} / B_{0 z}=10\right)$}

Figs. 80 plot for models $\mathrm{C} 1, \mathrm{C} 2$ and $\mathrm{C} 3$ the same quantities as Figs. 6.76 but under the assumption that the azimuthal magnetic field component is dominant $\left(B_{0 \phi} / B_{0 z}=10\right)$. In this case all models present monotonically unstable modes out to large radii ( $\sim \mathrm{Mpc})$. At all radii the unstable modes of model $\mathrm{C} 1$ have growth rate much higher than the cooling rate: the MRI dominates throughout the cool core, while in the cluster outskirts the unstable modes are driven by the MTI. Model C2 presents TI modes in the inner parts of the cool core, MRI modes in the intermediate radial range $(10-100 \mathrm{kpc})$ and MTI modes in the outskirts. Model C3, which has the lowest rotation speed at all radii (Fig. 3) is stable against MRI even when $B_{0 \phi}$ is dominant: its unstable modes are driven by TI in the cool core and by MTI in the outer regions where the temperature gradient is negative. In the explored models, both the MRI and the MTI modes have remarkably short timescales, of the order of a few local dynamical times. In absolute terms the MRI modes are fastest with growth times as short as $\sim 10^{8} \mathrm{yr}$ in the core.

Comparing Figs. 80 9 with Figs. 6 [7 it is apparent that the instabilities are stronger and more widespread when the magnetic field is dominated by the azimuthal component. The driving factor in determining these differences is not the presence of an azimuthal magnetic field component, but the fact that when $B_{0 \phi}$ is dominant, the vertical component of the magnetic field is weaker (at fixed $B_{0}$ ). As pointed out above, the MRI and the MTI 


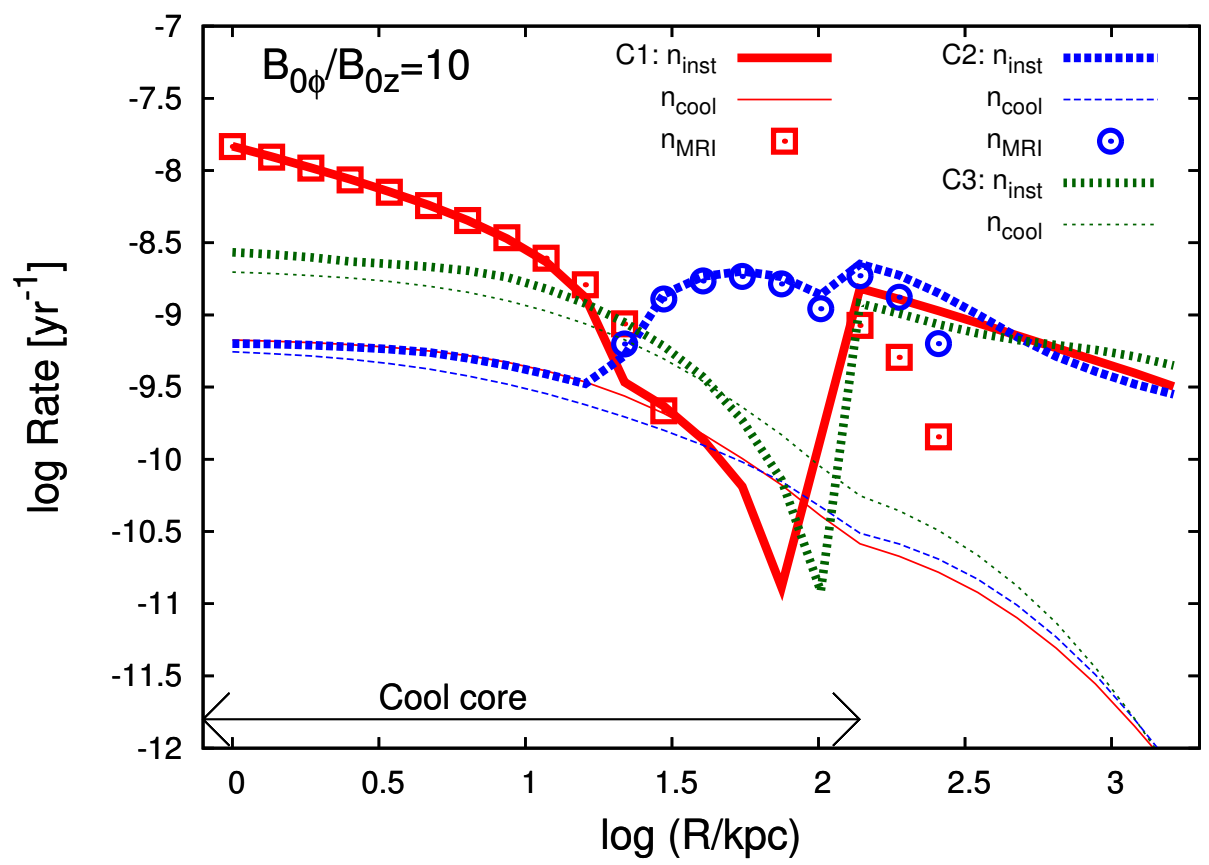

FIGURE 8. Maximum instability growth rate $n_{\text {inst }}$, cooling rate $n_{\text {cool }}$, and MRI growth rate $n_{\text {MRI }}$ as functions of radius in the equatorial plane of the same models as in Fig. 11 Here $B_{0 R}=0$, $B_{0 z} \neq 0$ and $B_{0 \phi} / B_{0 z}=10$.

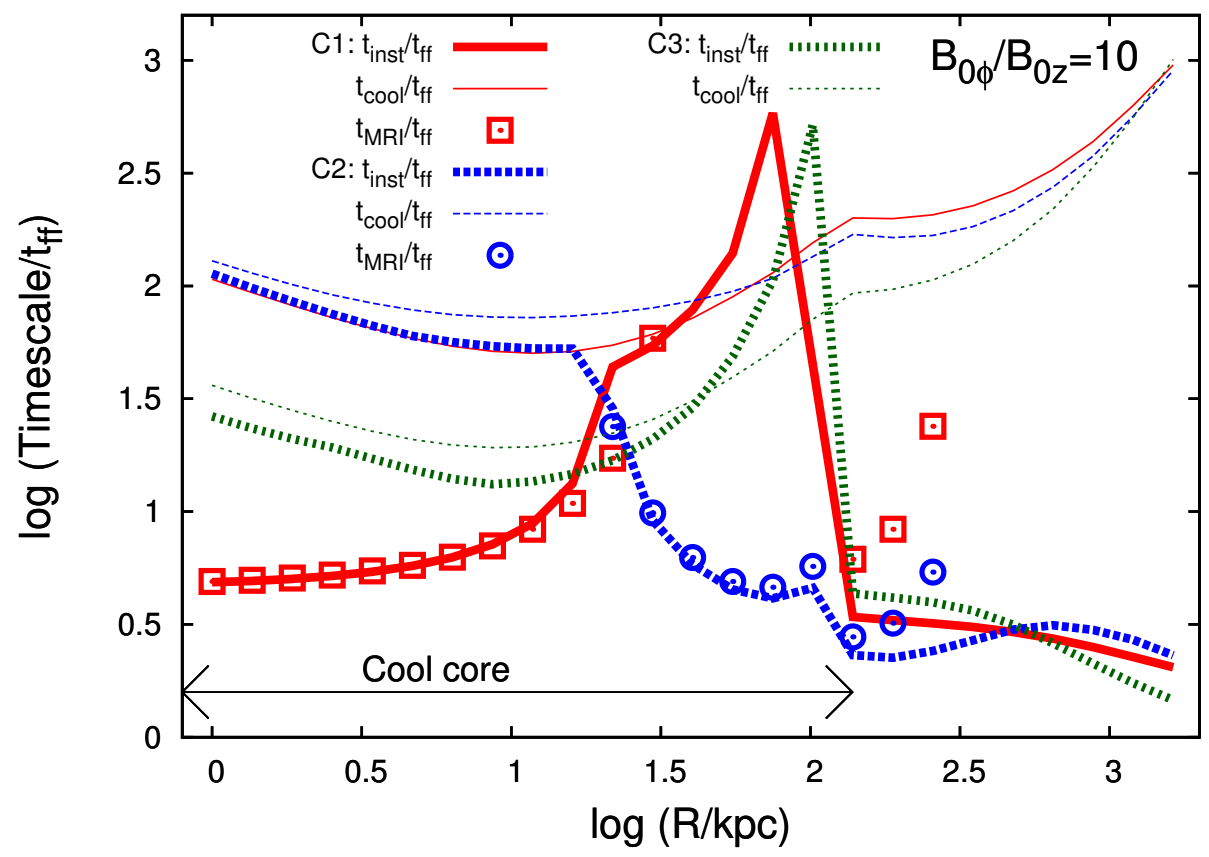

FIGURE 9 . Instability $\left(t_{\text {inst }}\right)$, cooling $\left(t_{\text {cool }}\right)$ and MRI $\left(t_{\mathrm{MRI}}\right)$ timescales normalized to the free-fall time $t_{\mathrm{ff}}$ as functions of radius in the equatorial plane of the same models as in Fig. 8 
occur only for wave-numbers smaller than critical values ( $k_{\mathrm{MRI}}$ and $k_{\mathrm{MTI}}$, respectively). Both $k_{\mathrm{MRI}}$ and $k_{\mathrm{MTI}}$ are inversely proportional to the magnetic field component coupled with the wave-vector (Balbus \& Hawlev 1991; Balbus 2000), which in the present case is just $B_{0 z}$. When the azimuthal magnetic field is dominant, the vertical magnetic field is sufficiently weak to trigger the MRI throughout the cores of models $\mathrm{C} 1$ and $\mathrm{C} 2$, and the MTI in the outer regions of all models. It is also the case that $k_{\mathrm{MRI}}$ increases for increasing angular velocity gradient, which explains why the MRI manifests itself in models $\mathrm{C} 1$ and $\mathrm{C} 2$, which have stronger angular velocity gradients than model C3 (see Fig. (4). When present, both the MRI and the MTI have growth rates much higher than the local cooling rate: the fastest instability is the MRI, which acts on timescales $10^{8}-10^{9} \mathrm{yr}$.

\section{Conclusions}

Building upon the results of NP13, NP14 and BEN13, in this paper we have studied local instabilities arising from axisymmetric perturbations in rotating cool-core clusters. For the sake of simplicity we limited ourselves to somewhat idealized cluster models. Notwithstanding these limitations, our calculations should catch the essential properties of more realistic cluster models that could be built in the near future, especially when more observational constraints on the ICM rotation are available, thanks to the nextgeneration X-ray missions such as ASTRO-H and ATHENA.

The results of the linear stability analysis of the rotating cool-core cluster models here presented show that, depending on the ICM properties, TI, MTI and MRI can occur at different radii in the equatorial plane (where, by construction, our models exclude the HBI, which however can play a role in more general configurations). What are the astrophysical consequences of these instabilities?

In principle, monotonically unstable TI modes can lead to local condensation of cold gas out of the hot plasma and therefore to a multiphase ICM. However, given that the growth rate of TIs is of the order of the cooling rate, the evolution of thermally unstable modes depend on the details of the balance of heating and cooling in the unperturbed fluid. While heat conduction always tend to damp thermal perturbations, other heating mechanisms such as AGN feedback have more complex effects and can in some cases even favour cooling instabilities (e.g. Ciotti \& Ostriker 2007; Gaspari et al. 2012). Therefore, it is not possible to draw robust conclusions on condensational modes based on the models here considered, in which we have adopted a very simple ad hoc heating source to balance radiative losses in the background plasma. Multiphase plasma is detected in cluster cool cores (Heckman 1981), but the origin of the colder medium is still debated. The observed correlation between presence of multiphase gas and short cooling time (low entropy) in cluster cores (e.g. Voit \& Donahue 2015) does not necessarily imply that the cold gas is produced by TI (Nipoti \& Binney 2004).

As far as the MTI is concerned, our results confirm previous findings that the MTI can be the dominant instability out of the cluster core. As in non-rotating ICM models, the MTI can affect the outer regions of the cluster (where the temperature decreases outwards) on times scales as short as a few local dynamical times, potentially driving strong turbulence in the non-linear regime (McCourt et al. 2011). We recall that our models do not account self-consistently for anisotropic momentum transport, which in principle can affect the behaviour of the MTI. However, it has been shown that, at least in non-rotating systems, the effects of the Braginskii viscosity, which can be significant for the HBI (Latter \& Kunz 2012), are almost negligible for the MTI (Kunz et al. 2012).

The most interesting instabilities among those found in this work are the MRI modes in the cool cores. Figs. 6. 9 show that the MRI rates are remarkably high, with associated 
timescales $10^{8}-10^{9} \mathrm{yr}$ (a few local dynamical times): this suggest that the MRI can have an important impact on cool cores. The MRI timescales are substantially shorter than the cooling time, so this conclusion is robust against uncertainties on the balance of heating and cooling in the core. The Braginskii viscosity, which is neglected in our calculations, could even enhance the growth rates of these dominant MRI modes (Quataert et al. 2002; Balbus 2004). Studies of weakly magnetized accretion discs (Balbus et al. 1994) have shown that the MRI is expected to drive turbulence, with associated energy and angular momentum transport. These MRI-driven dissipative processes can contribute substantially to the dynamics and evolution of cluster cool cores, if they rotate significantly. The MRI could be the engine of turbulent heating, which is believed to be an efficient mechanism to halt cooling flows in galaxy clusters (Zhuravleva et al. 2014). A natural development of the present work will be a quantitative estimate of the effect of the MRI on the energy balance and accretion rate of the plasma in cluster cool cores.

We acknowledge helpful discussions with Steven Balbus and Richard Lovelace. We thank the two anonymous referees for useful comments. CN has been supported by PRIN MIUR 2010-2011, project "The Chemical and Dynamical Evolution of the Milky Way and Local Group Galaxies", prot. 2010LY5N2T.

\section{Appendix A. Governing equations in cylindrical coordinates}

In cylindrical coordinates the governing equations (2.1) 2.4) read

$$
\begin{aligned}
& \frac{\partial \rho}{\partial t}+\frac{1}{R} \frac{\partial R \rho v_{R}}{\partial R}+\frac{1}{R} \frac{\partial \rho v_{\phi}}{\partial \phi}+\frac{\partial \rho v_{z}}{\partial z}=0, \\
& \frac{\partial v_{R}}{\partial t}+\mathbf{v} \cdot \nabla v_{R}-\frac{v_{\phi}^{2}}{R}=-\frac{1}{\rho} \frac{\partial p}{\partial R}-\frac{\partial \Phi}{\partial R}+\frac{1}{4 \pi \rho}\left(\mathbf{B} \cdot \nabla B_{R}-\frac{B_{\phi}^{2}}{R}\right)-\frac{1}{8 \pi \rho} \frac{\partial B^{2}}{\partial R}, \\
& \frac{\partial v_{\phi}}{\partial t}+\mathbf{v} \cdot \nabla v_{\phi}+\frac{v_{R} v_{\phi}}{R}=-\frac{1}{\rho R} \frac{\partial p}{\partial \phi}+\frac{1}{4 \pi \rho}\left(\mathbf{B} \cdot \nabla B_{\phi}+\frac{B_{R} B_{\phi}}{R}\right)-\frac{1}{8 \pi \rho} \frac{1}{R} \frac{\partial B^{2}}{\partial \phi}, \\
& \frac{\partial v_{z}}{\partial t}+\mathbf{v} \cdot \nabla v_{z}=-\frac{1}{\rho} \frac{\partial p}{\partial z}-\frac{\partial \Phi}{\partial z}+\frac{1}{4 \pi \rho}\left(\mathbf{B} \cdot \nabla B_{z}\right)-\frac{1}{8 \pi \rho} \frac{\partial B^{2}}{\partial z}, \\
& \frac{\partial B_{R}}{\partial t}=\mathbf{B} \cdot \nabla v_{R}-\mathbf{v} \cdot \nabla B_{R}-(\nabla \cdot \mathbf{v}) B_{R}, \\
& \frac{\partial B_{\phi}}{\partial t}=\mathbf{B} \cdot \nabla v_{\phi}+\frac{B_{\phi} v_{R}}{R}-\mathbf{v} \cdot \nabla B_{\phi}-\frac{v_{\phi} B_{R}}{R}-(\nabla \cdot \mathbf{v}) B_{\phi}, \\
& \frac{\partial B_{z}}{\partial t}=\mathbf{B} \cdot \nabla v_{z}-\mathbf{v} \cdot \nabla B_{z}-(\nabla \cdot \mathbf{v}) B_{z}, \\
& \frac{p}{\gamma-1}\left(\frac{\partial}{\partial t}+\mathbf{v} \cdot \nabla\right) \ln \left(p \rho^{-\gamma}\right)=\frac{1}{R} \frac{\partial}{\partial R}\left[R \frac{\chi(T) B_{R}(\mathbf{B} \cdot \nabla) T}{B^{2}}\right]+\frac{1}{R} \frac{\partial}{\partial \phi}\left[\frac{\chi(T) B_{\phi}(\mathbf{B} \cdot \nabla) T}{B^{2}}\right] \\
& \quad+\frac{\partial}{\partial z}\left[\frac{\chi(T) B_{z}(\mathbf{B} \cdot \nabla) T}{B^{2}}\right]-\rho \mathcal{L}(T, \rho)+\mathcal{H}(R, z),
\end{aligned}
$$

where we have assumed that the gravitational potential is axisymmetric and we have used $\nabla \cdot \mathbf{B}=0$ in writing the three components (equations A5 A7) of the induction equation (2.3). 


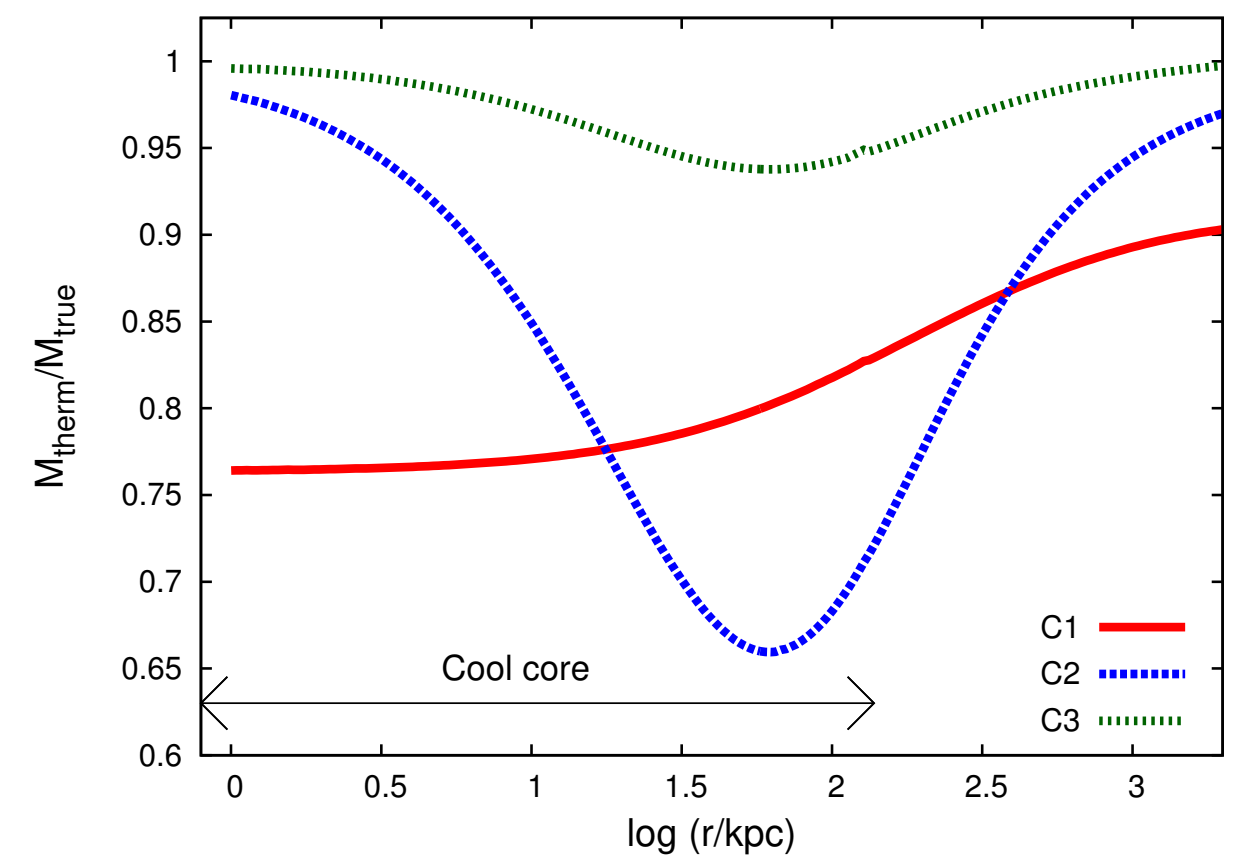

Figure 10 . Hydrostatic $\left(M_{\text {therm }}\right)$ to true $\left(M_{\text {true }}\right)$ mass ratio within a sphere of radius $r$ for the same models as in Fig. 1]

\section{Appendix B. Hydrostatic mass bias}

The estimate of the mass of galaxy clusters is mainly based on X-ray and gravitational lensing data. Mass estimates from X-rays are obtained in general under the assumption that the gas is in hydrostatic equilibrium, while mass estimates from gravitational lensing do not depend on the kinematics of gas. Deviations from hydrostatic equilibrium (due to rotation, bulk motions or turbulence) produce a discrepancy between the mass estimated from X-ray data and the mass estimated from gravitational lensing. Such a discrepancy, which is usually called hydrostatic mass bias, can be quantified by measuring within a sphere of radius $r$ the ratio $M_{\text {therm }}(r) / M_{\text {true }}(r)$, where $M_{\text {true }}$ is the true mass and $M_{\text {therm }}$ is the mass computed assuming that gravity is balanced only by the thermal-pressure gradient (Lau et al. 2013).

The ratio $M_{\text {therm }} / M_{\text {true }}$ is estimated in hydrodynamic cosmological simulations to be around $0.8-0.9$, with a slight variation with radius out to $r_{200}$ and a scatter of about 20 per cent (Meneghetti et al. 2010; Nelson et al. 2014). When X-ray simulated observations are used to mimic typical X-ray exposures, Rasia et al. (2012) show that temperature inhomogeneities could affect further the hydrostatic mass reconstruction by reducing $M_{\text {therm }} / M_{\text {true }}$ to $0.65-0.75$. On the observational side, we cannot probe the "true" mass but only a reasonable proxy for it as provided by gravitational lensing estimates. Programs like Weighing the Giants (WtG, von der Linden et al. 2014), CLASH (Donahue et al. 2014), and the Canadian Cluster Comparison Project (CCCP, Hoekstra et al. 2015) obtain values of the hydrostatic-to-lensing mass ratio in the range of $0.7-0.8$, although differences up to 40 per cent in either the weak lensing or X-ray mass measurement among different research groups are still measured (Sereno \& Ettori) 2015).

For models of rotating cluster such as C1, C2 and C3 (see Section 3) $M_{\text {therm }}<M_{\text {true }}$, 
because of the presence of rotational support. Models in which rotational support is more important are characterized by lower values of $M_{\text {therm }} / M_{\text {true }}$ : for a rotating model to be realistic, it must have $M_{\text {therm }} / M_{\text {true }}$ higher than the aforementioned observed values. It is therefore interesting to estimate the hydrostatic mass bias for models $\mathrm{C} 1, \mathrm{C} 2$ and $\mathrm{C} 3$. In all three cases $M_{\text {true }}(r)$ is just given by the spherically symmetric NFW mass distribution responsible for the total cluster potential (see Section 3). Following Lau et al. (2013), the hydrostatic mass profile can be computed as

$$
M_{\text {therm }}(r) \equiv-\frac{r^{2}}{G\left\langle\rho_{0}\right\rangle} \frac{\partial\left\langle p_{0}\right\rangle}{\partial r},
$$

where the symbol $\langle\cdots\rangle$ indicates an average over angles at fixed spherical radial coordinate $r$. We construct $\left\langle\rho_{0}\right\rangle$ and $\left\langle p_{0}\right\rangle$ by interpolating $\rho_{0}(R, Z)$ and $p_{0}(R, z)$ in spherical coordinates $(r, \theta)$ and then computing the average over $\theta$, at fixed $r$. The profiles of $M_{\text {therm }} / M_{\text {true }}$ for models C1, C2 and C3 are shown in Fig. 10. The minimum values of $M_{\text {therm }} / M_{\text {true }}$ are $0.76,0.66$ and 0.94 , for models C1, C2 and C3, respectively. However, these minima occur within the cool-core region $(r<100 \mathrm{kpc})$, in which the hydrostatic mass bias is not easily measured observationally. The minimum values of $M_{\text {therm }} / M_{\text {true }}$ in the radial range $r_{\mathrm{s}} \leqslant r \leqslant r_{200}$ (i.e. $0.5 \lesssim r / \mathrm{Mpc} \lesssim 2$ ), which is representative of the region typically probed by weak gravitational lensing, are $0.88(\mathrm{C} 1), 0.90(\mathrm{C} 2)$ and 0.98 (C3), consistent with the observational limits.

\section{REFERENCES}

Balbus, S. A. 2000 Stability, Instability, and "Backward" Transport in Stratified Fluids. ApJ 534, 420-427.

Balbus, S. A. 2001 Convective and Rotational Stability of a Dilute Plasma. ApJ 562, 909-917.

Balbus, S. A. 2004 Viscous Shear Instability in Weakly Magnetized, Dilute Plasmas. ApJ 616, 857-864.

Balbus, S. A., Gammie, C. F. \& Hawley, J. F. 1994 Fluctuations, dissipation and turbulence in accretion discs. MNRAS 271, 197.

Balbus, S. A. \& Hawley, J. F. 1991 A powerful local shear instability in weakly magnetized disks. I - Linear analysis. II - Nonlinear evolution. ApJ 376, 214-233.

Bianconi, M., Ettori, S. \& Nipoti, C. 2013 Gas rotation in galaxy clusters: signatures and detectability in X-rays. MNRAS 434, 1565-1575 (BEN13).

Binney, J., Nipoti, C. \& Fraternali, F. 2009 Do high-velocity clouds form by thermal instability? MNRAS 397, 1804-1815.

Braginskit, S. I. 1965 Transport Processes in a Plasma. Reviews of Plasma Physics 1, 205.

BrügGen, M. 2013 Magnetic fields in galaxy clusters. Astronomische Nachrichten 334, 543.

Chandrasekhar, S. 1960 The Stability of Non-Dissipative Couette Flow in Hydromagnetics. Proceedings of the National Academy of Science 46, 253-257.

Ciotti, L. \& Ostriker, J. P. 2007 Radiative Feedback from Massive Black Holes in Elliptical Galaxies: AGN Flaring and Central Starburst Fueled by Recycled Gas. ApJ 665, 10381056.

Donahue, M., Voit, G. M., Mahdavi, A., Umetsu, K., Ettori, S., Merten, J., Postman, M., Hoffer, A., Baldi, A., Coe, D., Czakon, N., Bartelmann, M., Benitez, N., Bouwens, R., Bradley, L., Broadhurst, T., Ford, H., Gastaldello, F., Grillo, C., Infante, L., Jouvel, S., Koekemoer, A., Kelson, D., Lahav, O., Lemze, D., Medezinski, E., Melchior, P., Meneghetti, M., Molino, A., Moustakas, J., Moustakas, L. A., Nonino, M., Rosati, P., Sayers, J., Seitz, S., Van der Wel, A., Zheng, W. \& Zitrin, A. 2014 CLASH-X: A Comparison of Lensing and X-Ray Techniques for Measuring the Mass Profiles of Galaxy Clusters. ApJ 794, 136.

Ettori, S., Pratt, G. W., de PlaA, J., Eckert, D., Nevalainen, J., Battistelli, E. S., Borgani, S., Croston, J. H., Finoguenov, A., Kaastra, J., Gaspari, M., Gastaldello, F., Gitti, M., Molendi, S., Pointecouteau, E., Ponman, T. J., 
Reiprich, T. H., Roncarelli, M., Rossetti, M., Sanders, J. S., Sun, M., Trinchieri, G., Vazza, F., Arnaud, M., Böringher, H., Brighenti, F., Dahle, H., De Grandi, S., Mohr, J. J., Moretti, A. \& Schindler, S. 2013 The Hot and Energetic Universe: The astrophysics of galaxy groups and clusters. ArXiv e-prints, arXiv:1306.2322 .

Fang, T., Humphrey, P. \& Buote, D. 2009 Rotation and Turbulence of the Hot Intracluster Medium in Galaxy Clusters. ApJ 691, 1648-1659.

Ferraro, V. C. A. 1937 The non-uniform rotation of the Sun and its magnetic field. MNRAS 97, 458.

Field, G. B. 1965 Thermal Instability. ApJ 142, 531.

Gaspari, M., Ruszkowski, M. \& Sharma, P. 2012 Cause and Effect of Feedback: Multiphase Gas in Cluster Cores Heated by AGN Jets. ApJ 746, 94.

HeCKMAn, T. M. 1981 Optical emission-line gas associated with dominant cluster galaxies. ApJ 250, L59-L63.

Hoekstra, H., Herbonnet, R., Muzzin, A., Babul, A., Mahdavi, A., Viola, M. \& CaCCiAto, M. 2015 The Canadian Cluster Comparison Project: detailed study of systematics and updated weak lensing masses. MNRAS 449, 685-714.

Kitayama, T., Bautz, M., Markevitch, M., Matsushita, K., Allen, S., Kawaharada, M., Mcnamara, B., Ota, N., Akamatsu, H., de Plaa, J., Galeazzi, M., Madejski, G., Main, R., Miller, E., Nakazawa, K., Russell, H., Sato, K., Sekiya, N., Simionescu, A., Tamura, T., Uchida, Y., Ursino, E., Werner, N., Zhuravleva, I., ZuHone, J. \& on Behalf of the ASTRO-H Science Working Group 2014 ASTRO-H White Paper - Clusters of Galaxies and Related Science. ArXiv e-prints, arXiv:1412.1176

Kunz, M. W. 2011 Dynamical stability of a thermally stratified intracluster medium with anisotropic momentum and heat transport. MNRAS 417, 602-616.

Kunz, M. W., Bogdanović, T., Reynolds, C. S. \& Stone, J. M. 2012 Buoyancy Instabilities in a Weakly Collisional Intracluster Medium. ApJ 754, 122.

Latter, H. N. \& Kunz, M. W. 2012 The HBI in a quasi-global model of the intracluster medium. MNRAS 423, 1964-1972.

Lau, E. T., Nagai, D., Kravtsov, A. V., Vikhlinin, A. \& Zentner, A. R. 2012 Constraining Cluster Physics with the Shape of X-Ray Clusters: Comparison of Local X-Ray Clusters Versus $\Lambda$ CDM Clusters. ApJ 755, 116.

Lau, E. T., Nagai, D. \& Nelson, K. 2013 Weighing Galaxy Clusters with Gas. I. On the Methods of Computing Hydrostatic Mass Bias. ApJ 777, 151.

Malagoli, A., Rosner, R. \& Bodo, G. 1987 On the thermal instability of galactic and cluster halos. ApJ 319, 632-636.

McCourt, M., Parrish, I. J., Sharma, P. \& Quataert, E. 2011 Can conduction induce convection? On the non-linear saturation of buoyancy instabilities in dilute plasmas. MNRAS 413, 1295-1310.

McCourt, M., Sharma, P., Quataert, E. \& Parrish, I. J. 2012 Thermal instability in gravitationally stratified plasmas: implications for multiphase structure in clusters and galaxy haloes. MNRAS 419, 3319-3337.

Meneghetti, M., Rasia, E., Merten, J., Bellagamba, F., Ettori, S., Mazzotta, P., DolaG, K. \& MARrI, S. 2010 Weighing simulated galaxy clusters using lensing and Xray. A\&A 514, A93.

Nagai, D., Lau, E. T., Avestruz, C., Nelson, K. \& Rudd, D. H. 2013 Predicting Mergerinduced Gas Motions in $\Lambda$ CDM Galaxy Clusters. ApJ 777, 137.

Navarro, J. F., Frenk, C. S. \& White, S. D. M. 1995 Simulations of X-ray clusters. MNRAS 275, 720-740.

Nelson, K., Lau, E. T., Nagai, D., Rudd, D. H. \& Yu, L. 2014 Weighing Galaxy Clusters with Gas. II. On the Origin of Hydrostatic Mass Bias in $\Lambda$ CDM Galaxy Clusters. ApJ 782, 107.

Nipoti, C. 2010 Thermal instability in rotating galactic coronae. MNRAS 406, 247-263.

Nipoti, C. \& Binney, J. 2004 Cold filaments in galaxy clusters: effects of heat conduction. MNRAS 349, 1509-1515.

Nipoti, C. \& Posti, L. 2013 Thermal stability of a weakly magnetized rotating plasma. MNRAS 428, 815-827 (NP13). 
Nipoti, C. \& Posti, L. 2014 On the Nature of Local Instabilities in Rotating Galactic Coronae and Cool Cores of Galaxy Clusters. ApJ 792, 21 (NP14).

Parrish, I. J., McCourt, M., Quataert, E. \& Sharma, P. 2012 The effects of anisotropic viscosity on turbulence and heat transport in the intracluster medium. MNRAS 422, 704718.

Parrish, I. J., Quataert, E. \& Sharma, P. 2009 Anisotropic Thermal Conduction and the Cooling Flow Problem in Galaxy Clusters. ApJ 703, 96-108.

Pinto, C., Sanders, J. S., Werner, N., de PlaA, J., Fabian, A. C., Zhang, Y.-Y., KaAstra, J. S., Finoguenov, A. \& Ahoranta, J. 2015 Chemical Enrichment RGS cluster Sample (CHEERS): Constraints on turbulence. A\&A 575, A38.

QuATAert, E. 2008 Buoyancy Instabilities in Weakly Magnetized Low-Collisionality Plasmas. ApJ 673, 758-762.

Quataert, E., Dorland, W. \& Hammett, G. W. 2002 The Magnetorotational Instability in a Collisionless Plasma. ApJ 577, 524-533.

Rasia, E., Meneghetti, M., Martino, R., Borgani, S., Bonafede, A., Dolag, K., Ettori, S., Fabjan, D., Giocoli, C., Mazzotta, P., Merten, J., Radovich, M. \& TorNATORE, L. 2012 Lensing and x-ray mass estimates of clusters (simulations). New Journal of Physics 14 (5), 055018.

SAnders, J. S. \& FABIAn, A. C. 2013 Velocity width measurements of the coolest X-ray emitting material in the cores of clusters, groups and elliptical galaxies. MNRAS 429, $2727-2738$.

Sereno, M. \& EtTori, S. 2015 Comparing Masses in Literature (CoMaLit)-I. Bias and scatter in weak lensing and X-ray mass estimates of clusters. MNRAS, in press (arXiv:1407.7868v2) .

SpItzer, L. 1962 Physics of Fully Ionized Gases. New York: Interscience (2nd edition).

Sutherland, R. S. \& Dopita, M. A. 1993 Cooling functions for low-density astrophysical plasmas. ApJS 88, 253-327.

Takahashi, T., Mitsuda, K., Kelley, R., Fabian, A., Mushotzky, R., Ohashi, T., Petre, R. \& on Behalf of the ASTRO-H Science Working Group 2014 ASTRO-H White Paper - Introduction. ArXiv e-prints, arXiv:1412.2351.

Velikhov, E. P. 1959 Stability of an ideally conducting liquid flowing between cylinders rotating in a magnetic field. Soviet Phys. JETP 9, 995.

Voit, G. M. \& Donahue, M. 2015 Cooling Time, Freefall Time, and Precipitation in the Cores of ACCEPT Galaxy Clusters. ApJ 799, L1.

von der linden, A., Mantz, A., Allen, S. W., Applegate, D. E., Kelly, P. L., Morris, R. G., Wright, A., Allen, M. T., Burchat, P. R., Burke, D. L., Donovan, D. \& EBELING, H. 2014 Robust weak-lensing mass calibration of Planck galaxy clusters. MNRAS 443, 1973-1978.

Zhuravleva, I., Churazov, E., Scherochinin, A. A., Allen, S. W., Arévalo, P., Fabian, A. C., Forman, W. R., Sanders, J. S., Simionescu, A., Sunyaev, R., Vikhlinin, A. \& Werner, N. 2014 Turbulent heating in galaxy clusters brightest in X-rays. Nature 515, $85-87$. 